\title{
Knockdown of Long Non-coding RNA UCA1 Represses Gallbladder Cancer Advancement by Regulating SPOCK1 Expression via Sponging miR- 613
}

\section{Tao Zhang}

Loudi Central Hospital of Hunan

Lijian Chen

Hunan Children's Hospital

\section{Xundi Xu}

the Second Xiangya Hospital of Central South Hospital

Chao Shen ( $\nabla$ xlxwbj@163.com )

Loudi Central Hospital of Hunan

\section{Research}

Keywords: GBC, UCA1, miR-613, SPOCK1

Posted Date: July 20th, 2020

DOI: https://doi.org/10.21203/rs.3.rs-42724/v1

License: (c) (1) This work is licensed under a Creative Commons Attribution 4.0 International License.

Read Full License 


\section{Abstract}

OBJECTIVE : Patients with advanced gallbladder cancer (GBC) have a lower 5-year survival rate. Long non-coding RNA urothelial carcinoma associated 1 (UCA1) and miR-613 are involved in the progression of various cancers. This study was to explore the regulatory mechanism between UCA1 and miR-613 in GBC.

METHODS: The expression levels of UCA1, miR-613, and SPOCK1 mRNA were detected using qRT-PCR. Cell proliferation, migration, invasion, and apoptosis were determined with MTT, transwell, or flow cytometry assays. The levels of SPOCK1 protein, Bax, cleaved-casp-3, and Bcl-2 were determined by western blot analysis. The relationship between miR-613 and UCA1 or SPOCK1 was verified via dualluciferase reporter and/or RNA immunoprecipitation (RIP) assays. The role of UCA1 in vivo was confirmed by xenograft assay.

RESULTS: UCA1 and SPOCK1 were upregulated while miR-613 was downregulated in GBC tissues and cells. UCA1 silencing blocked tumor growth in vivo, impeded cell proliferation, migration, invasion, and induced cell apoptosis in GBC cells in vitro. Notably, UCA1 acted as a sponge for miR-613, which targeted SPOCK1 in GBC cells. Moreover, miR-613 repressed cell proliferation, migration, invasion, and accelerated cell apoptosis in GBC cells. UCA1 enhancement reversed miR-613 mimic-mediated influence on proliferation, migration, invasion, and apoptosis of GBC cells. UCA1 regulated SPOCK1 expression through miR-613. Furthermore, SPOCK1 elevation overturned UCA1 silencing-mediated the malignant behaviors of GBC cells.

CONCLUSION: UCA1 knockdown suppressed GBC progression via downregulating SPOCK1 via sponging miR-613, providing an evidence for UCA1 as a target for GBC treatment.

\section{Highlights}

1. UCA1 and SPOCK1 were upregulated in GBC tissues and cells.

2. MiR-613 expression was downregulated in GBC tissues and cells.

3. Inhibition of UCA1 impeded tumor growth in vivo, blocked cell proliferation, migration, invasion, and induced cell apoptosis in GBC cells.

4. UCA1 was verified as a sponge for miR-613 in GBC cells.

5. SPOCK1 served as a target for miR-613 in GBC tissues.

\section{Introduction}

Gallbladder cancer (GBC), which originates from biliary tract cells, is a malignant tumor [1]. The 5-year survival rate of advanced GBC is only about $5 \%$, which is mainly due to the low specificity of tumor diagnosis, which leads to poor treatment prognosis $[2,3]$. Therefore, there is an urgent need to further 
study the molecular mechanism of GBC development in order to provide effective targets for the diagnosis and treatment of GBC.

Long non-coding RNAs (IncRNAs), a classes of transcripts that do have protein-coding potential, are important regulatory factors in a variety of biological processes [4]. Numerous researches have shown that the dysregulation of IncRNAs may be related to the advancement of cancers [5]. Long non-coding RNA Urothelial carcinoma associated 1 (UCA1), an oncofoetal gene, which was primitively found in human bladder cancer cells. UCA1 was reported to be involved in the development of various cancers [68]. For instance, Nie et al. indicated that UCA1 regulated ERBB4 expression via sponging miR-193a-3p, which contributed to the progression of non-small cell lung cancer [6]. Previous study manifested that UCA1 was associated with the development of GBC [9]. However, the regulatory mechanism of UCA1 in $\mathrm{GBC}$ is still unclear.

MicroRNAs (miRNAs) are a type of short non-coding RNAs that regulate gene expression via binding to the $3^{\prime}$ untranslated region (UTR) of target mRNAs [10]. Studies have shown that miRNAs play a crucial role in human physiological and pathological processes $[11,12]$. A large amount of miRNAs have been proved as potential diagnostic biomarkers and therapeutic targets for various cancers $[13,14]$. MicroRNA613 (miR-613) was demonstrated to play important role in cancer progression. For instance, miR-613 suppressed tumor progression in triple-negative breast cancer [15], bladder cancer [16], and laryngeal squamous cell cancer [17]. To date, the role of miR-613 in GBC has rarely been reported.

Cysteine-rich acidic secreted protein/osteonectin, cwcv, and kazal-like domains proteoglycan 1 (SPOCK1), a member of $\mathrm{ca}^{2+}$-binding proteoglycan family, is involved in cell proliferation, adhesion, and migration [18]. A series of studies have shown that SPOCK1 acted as an oncogene in multiple cancers [19-21]. In addition, Shu et al. reported that SPOCK1 was associated with the development of GBC [22]. Up to now, it is unclear whether SPOCK1 can be regulated by UCA1 and miR-613 in GBC.

Hence, the study aimed to explore the role and molecular mechanism of UCA1 in GBC, and the results manifested that UCA1 knockdown impeded GBC progression through the miR-613/SPOCK1 axis.

\section{Materials And Methods}

\section{GBC samples}

30 paired GBC tissue samples and adjacent normal tissue samples were collected from Loudi Central Hospital of Hunan. The histology and pathology of fresh samples were verified by at last two pathologists. GBC patients did not receive chemotherapy, radiotherapy, or other anticancer drugs before surgery. This study was approved by the ethics committee of Loudi Central Hospital of Hunan. All patients signed the informed consents before undergoing surgical resection.

\section{Cell culture and transfection}


Human GBC cell lines (SGC-996, GBC-SD, and NOZ) and human intrahepatic bile duct epithelial cells (HIBEpiC) were used in the study. SGC-996, GBC-SD, and HIBEpiC cells were obtained from Jingkang Biological Engineering Co., Ltd. (Shanghai, China). NOZ cells were obtained from Bena culture collection (Suzhou, China). Roswell Park Memorial Institute (RPMI) 1640 medium (Thermo Fisher Scientific, Waltham, MA, USA) supplemented with 10\% fetal bovine serum (FBS) (Thermo Fisher Scientific) and 100 $\mathrm{U} / \mathrm{mL}$ penicillin/streptomycin (Corning Inc., Corning, NY, USA) was used to culture all cell lines. All cell lines were incubated in an atmosphere with $5 \% \mathrm{CO}_{2}$ at $37^{\circ} \mathrm{C}$.

Short hairpin RNA (sh-RNA) targeting UCA1 (sh-UCA1) and negative control (sh-NC) were synthesized by Genepharma (Shanghai, China). The sequences of UCA1 and SPOCK1 were cloned into the pcDNA3.1 vector (vector) (Invitrogen, Carlsbad, CA, USA) to construct the overexpression vectors for UCA1 and SPOCK1, respectively. MiR-613 mimic and inhibitor (miR-613 and anti-miR-613) and their corresponding negative controls (miR-NC and anti-miR-NC) were purchased from Genepharma. Oligonucleotides or plasmids were transfected into NOZ and GBC-SD cells using the lipofectamine 2000 reagent (Thermo Fisher Scientific).

\section{Quantitative real-time polymerase chain reaction (qRT-PCR)}

Total RNA was extracted from GBC tissues and cells by TRIzol reagent (Transgen Biotech, Beijing, China). Reverse transcription was executed through using All-in-OneTM miRNA First stand cDNA Synthesis Kit (GeneCopoeia, Rockville, MD, USA) or Prime Script ${ }^{T M}$ RT reagent kit (Takara, Shiga, Japan). Real time PCR was performed by using Platinum SYBR Green qPCR SuperMix UDG (Invitrogen). The primers were listed as below: UCA1 (forward, 5'-CTCTCCATTGGGTTCACCATTC-3'; reverse, 5'-

GCGGCAGGTCTTAAGAGATGAG-3'), miR-613 (forward, 5'-CGTTTC TTCCTTGTAAGGA-3'; reverse, 5'CCCAAGCTTGTTGGAGAACAGCAGCGAGGAC-3'), and SPOCK1 (forward, 5'-CACTGGGTTGGACCTTCGA-3'; reverse, 5'-CTTTGGTGGCTCAGGCTCT-3'), glyceraldehyde-3-phosphate dehydrogenase (GAPDH) (forward, 5'-GACTCCACTCACGGCAAATTCA-3'; reverse, 5'-TCGCTCCTGGAAGATGGTGAT-3'), and U6 small nuclear RNA (snRNA) (Forward: 5'-GCTCGCTTCGGCAGCACA-3' and Reverse: 5'-GAGGTATTCGCACCAGAGGA-3'). GAPDH served as an internal reference gene for UCA1 and SPOCK1. U6 snRNA was viewed as an internal control for miR-613. Relative expression was calculated by using the $2^{-\Delta \Delta \mathrm{Ct}}$ method.

\section{Western blot analysis}

Total protein of GBC tissues and cells was extracted using RIPA protein extraction reagent (Beyotime, Shanghai, China). Total protein were isolated by electrophoresis on $10 \%$ SDS-PAGE and transferred onto the PVDF membranes (Millipore, Billerica, MA, USA). The PVDF membranes were blocked in TBST buffer with $5 \%$ non-fat milk for $1 \mathrm{~h}$. Then, the membranes were incubated with primary antibodies: anti-cleaved caspase-3 (ab32042, 1:1,000, Abcam, Cambridge, MA, USA), anti-Bax (ab182733, 1:1000, Abcam), antiBcl-2 (ab32124, 1:1000, Abcam), anti-SPOCK1 (ab229935, 1:2000, Abcam), and anti-GAPDH (ab9485, 1:1000, Abcam). Subsequently, the membranes were incubated with secondary antibodies: goat antirabbit IgG (ab6721, 1:10000, Abcam). The immunoblot was visualized through the enhanced chemiluminescence solution (Beyotime). 


\section{Cell proliferation assay}

The proliferation of NOZ and GBC-SD cells was evaluated by the 3-(4,5-dimethylthiazol-2-yl)-2,5diphenyltetrazolium bromide (MTT) kit (Sigma, St. Louis, Mo, USA). Briefly, the transfected NOZ and GBCSD cells ( 5000 cells/well) were seeded into 96 -well plates for $24 \mathrm{~h}, 48 \mathrm{~h}$, and $72 \mathrm{~h}$. Then, MTT (20 uL, $5 \mathrm{mg} / \mathrm{mL}$ ) was added to each well and kept for $4 \mathrm{~h}$. Next, DMSO (200 uL) was added for the dissolution of the formazan crystals. The optical density (OD) at $490 \mathrm{~nm}$ was measured using a Microplate Reader (Thermo Fisher Scientific).

\section{Transwell assay}

The migration of transfected NOZ and GBC-SD cells was evaluated via the transwell chamber $(8 \mu \mathrm{m}, \mathrm{BD}$ Biosciences, San Jose, CA, USA). Briefly, the transfected NOZ and GBC-SD cells $\left(5 \times 10^{4}\right)$ were seeded to the upper of the chamber with RPMI 1640 medium. And the lower of the chamber was supplemented with RPMI 1640 containing FBS (10\%). After culture for $24 \mathrm{~h}$, the cells on the lower surface of the membrane were fixed via paraformaldehyde (4\%, Sigma) and stained with crystal violet $(0.1 \%$, Solarbio, Beijing, China). The transwell chamber coated with matrigel matrix (BD Biosciences) was used for invasion assay. In the end, the inverted microscope (Olympus, Tokyo, Japan) was applied to count the migrated or invaded cells.

\section{Cell apoptosis assay}

The apoptotic rate of transfected NOZ and GBC-SD cells was determined by the Annexin V-fluorescein isothiocyanate (FITC)/PI apoptosis detection kit (BD Biosciences). Briefly, after transfection for $48 \mathrm{~h}$, the transfected NOZ and GBC-SD cells $\left(1 \times 10^{5}\right)$ were resuspended in binding solution and then stained with annexin V-FITC and PI. After 20 min incubation in the dark, the apoptotic rate was determined using a FACScan ${ }^{\circledR}$ flow cytometry (BD Biosciences).

\section{Dual-luciferase reporter assay}

The starBase2.0 database was used to predict the binding sites between miR-613 and UCA1 or SPOCK1. The sequences of wild type UCA1 (WT-UCA1), mutant UCA1 (MUT-UCA1), wild type SPOCK1 (SPOCK1 3'UTR-WT), mutant SPOCK1 (SPOCK1 3'UTR-MUT) containing putative binding sites for miR-613 were synthesized by Geneseed Biotech Co., Ltd (Guangzhou, China). NOZ and GBC-SD cells were cotransfected with luciferase reporter vectors and miR-613 or miR-NC by Lipofectamine 2000 reagent, respectively. The luciferase activity was measured using luciferase reporter assay kit (Promega) after transfection for $48 \mathrm{~h}$.

\section{RNA immunoprecipitation (RIP) assay}

The relationship between UCA1 and miR-613 was verified via the EZ-Magna RIP RNA-Binding Protein Immunoprecipitation Kit (Millipore, Billerica, MA, USA). NOZ and GBC-SD cells were lysed with RIP lysis buffer. Following this, the lysates were incubated with the RIP buffer containing magnetic beads conjugated with anti-IgG or anti-Ago2 antibodies (Millipore). Subsequently, the magnetic beads were 
treated with proteinase K (Sigma). The relative enrichment of UCA1 and miR-613 was analyzed by qRTPCR.

\section{Xenograft assay}

10 BALB/c nude mice (athymic, 3-week-old) were acquired from Shanghai Experimental Animal Center (Shanghai, China). The animal experiment was ratified by the Animal Ethics Committee of Loudi Central Hospital of Hunan. In short, NOZ cells $\left(1 \times 10^{6}\right)$ with sh-NC or lentivirus-mediated sh-UCA1 (RiboBio) were subcutaneously injected into the dorsal side of the nude mice ( 5 mice/group). Tumor volume was figured through the equation: Volume $=\left(\right.$ length $\times$ width $\left.^{2}\right) / 2$. Tumor volume was measured every 5 days until day 35. After 5 weeks, the mice were euthanized for subsequent analysis.

\section{Statistical analysis}

The experiments in vitro were repeated at the least 3 times. GraphPad Prism 5.0 (GraphPad, San Diego, CA, USA) and SPSS 22.0 software (SPSS, Chicago, IL, USA) were utilized for statistical analysis. Data were exhibited as mean \pm standard deviation. Person's correlation analysis was employ to determine the correlation between UCA1 and miR-613. Comparisons of between 2 or among more groups were determined by Student's $t$ test or one-way variance analysis (ANOVA). Differences with $P<0.05$ were statistically significant.

\section{Results}

UCA1 was upregulated and miR-613 was downregulated in GBC tissues and cells.

In the first place, we detected the expression of UCA1 and miR-613 in 30 pairs of GBC and adjoining normal tissues through qRT-PCR. The results presented that UCA1 expression was obviously upregulated in GBC tissues when compared to the adjoining normal tissues, while the expression of miR-613 had the opposite trend (Fig. 1A and 1B). Moreover, the expression of UCA1 was negatively correlated with miR613 in GBC tissues (Fig. 1C). Compared with the HIBEpic cells, UCA1 expression was signally upregulated while miR-613 expression was markedly downregulated in GBC cells (SGC-996, GBC-SD, and NOZ) (Fig. 1D and 1E). Therefore, these results indicated that the aberrant expression of UCA1 and miR-613 might be involved in the development of GBC.

\section{Knockdown of UCA1 suppressed cell proliferation, migration, invasion, and promoted cell apoptosis in GBC cells}

In consideration of the above results, we verified the role of UCA1 in GBC through loss-of-function experiments. Firstly, the results of qRT-PCR presented that UCA1 expression was obviously decreased in NOZ and GBC-SD cells transfected with sh-UCA1 in comparison to the control sh-NC (Fig. 2A). Moreover, MTT assays indicated that the proliferation of NOZ and GBC-SD cells were markedly repressed by UCA1 
downregulation in comparison with the control group (Fig. 2B and 2C). Transwell assays manifested that the migration and invasion of UCA1-silenced NOZ and GBC-SD cells were curbed (Fig. 2D-2G). Flow cytometry assays disclosed that the apoptotic rate of NOZ and GBC-SD cells was conspicuously higher in UCA1-suppressed NOZ and GBC-SD cells (Fig. $2 \mathrm{H}$ and 2I). Also, western blot analysis exhibited that UCA1 silencing downregulated the levels of Bax and cleaved-casp-3 proteins and upregulated the level of Bcl-2 in NOZ and GBC-SD cells (Fig. 2J and 2K). These findings suggested that UCA1 knockdown inhibited cell proliferation, migration, invasion, and facilitated cell apoptosis in GBC cells.

\section{UCA1 severed as a sponge for miR-613 in GBC cells}

To investigate the targeting relationship between UCA1 and miR-613 in GBC, we first predicted the potential binding sits between UCA1 and miR-613 through an online bioinformatics database starBase2.0. The results exhibited that miR-613 had complementary bases paring with UCA1 (Fig. 3A). Subsequently, dual-luciferase reporter assay showed that miR-613 overexpression prominently impeded the luciferase activity of WT-UCA1 in NOZ and GBC-SD cells relative to the control group (Fig. 3B and 3C). Moreover, RIP assay presented that UCA1 and miR-613 were conspicuously enriched in Ago2-containing beads than that in IgG-harboring beads (Fig. 3D and 3E). Also, the expression of UCA1 was elevated in NOZ and GBC-SD cells transfected with UCA1 in comparison to the control vector (Fig. 3F). In addition, miR-613 expression was repressed after UCA1 transfection and increased by UCA1 knockdown in NOZ and GBC-SD cells (Fig. 3G and $3 \mathrm{H}$ ). Collectively, these results manifested that miR-613 served as a target for UCA1 in GBC cells.

UCA1 enhancement restored miR-613 upregulation-mediated effects on the proliferation, migration, invasion, and apoptosis of GBC cells

Next, we further explored the influence of UCA1 overexpression on the proliferation, migration, invasion, and apoptosis of GBC cells mediated by miR-613 mimic. QRT-PCR manifested that miR-613 expression was prominently increased in NOZ and GBC-SD cells transfected with miR-613 that that the control group (Fig. 4A). MTT assays showed that miR-613 overexpression repressed the proliferation of NOZ and GBC-

SD cells, while this influence was recovered by UCA1 elevation (Fig. 4B). In addition, the repressive effect of miR-613 upregulation on the migration and invasion of NOZ and GBC-SD cells was overturned by UCA1 overexpression (Fig. 4C and 4D). Besides, the apoptotic rate of miR-613-enhanced NOZ and GBCSD cells was increased, while this elevation was abolished by UCA1 overexpression (Fig. 4E). In addition, the upregulation of Bax and cleaved-casp-3 and the downregulation of Bcl-2 in NOZ and GBC-SD cells caused by miR-613 mimic were restored by UCA1 overexpression (Fig. 4F and 4G). These results showed that UCA1 regulated cell proliferation, migration, invasion, and apoptosis in GBC cells via miR-613.

\section{SPOCK1 was verified as a target for miR-613 in GBC cells}

In view of the above findings, we proposed that UCA1 might exert its function via acting as a ceRNA to regulate the target of miR-613. As shown in Fig. 5A, SPOCK1 was predicted as a target for miR-613. The 
results of dual-luciferase reporter assays showed that the luciferase activity of SPOCK1 3'UTR-WT in NOZ and GBC-SD cells transfected with miR-613 was effectively attenuated compared with the mi-NC, but there was no remarkable difference in the luciferase intensity of SPOCK1 3'UTR-MUT (Fig. 5B and 5C). Also, compared to the control anti-miR-NC, miR-613 expression was reduced in NOZ and GBC-SD cells transfected with anti-miR-613 (Fig. 5D). Furthermore, the level of SPOCK1 protein was obviously inhibited by miR-613 mimic and elevated by miR-613 knockdown in NOZ and GBC-SD cells (Fig. 5E and 5F). Additionally, SPOCK1 mRNA and protein levels were drastically upregulated in GBC tissues and cells (Fig. 5G-5I). Taken together, SPOCK1 served as a downstream target for miR-613 in GBC cells.

\section{SPOCK1 overexpression reversed UCA1 inhibition-mediated effects on the proliferation, migration, invasion, and apoptosis of GBC cells}

Given that UCA1 acted as a sponge for miR-613, which targeted SPOCK1 in GBC cells. We surveyed whether UCA1 exerted its function via SPOCK1 in GBC cells. Firstly, silenced UCA1 expression downregulated the level of SPOCK1 protein in NOZ and GBC-SD cells, but this repression was abrogated by miR-613 inhibitor (Fig. 6A and 6B). Moreover, the level of SPOCK1 protein was elevated in NOZ and GBC-SD cells transfected with SPOCK1 compared with the negative control vector (Fig. 6C). MTT assays presented that overexpression of SPOCK1 overturned the repression of proliferation of NOZ and GBC-SD cells caused by UCA1 silencing (Fig. 6D and 6E). Transwell assays manifested that elevated SPOCK1 expression overturned the suppressive effect of UCA1 knockdown on the migration and invasion of NOZ and GBC-SD cells (Fig. 6F and 6G). Flow cytometry assays showed that the facilitation of apoptosis of NOZ and GBC-SD cells mediated by UCA1 knockdown was abolished by SPOCK1 elevation (Fig. 6H). Furthermore, the upregulation of SPOCK1 reversed UCA1 knockdown-mediated effects on the levels of Bax, cleaved-casp-3, and Bcl-2 in NOZ and GBC-SD cells (Fig. $6 \mathrm{I}$ and $6 \mathrm{~J}$ ). Collectively, these results showed that UCA1 modulated cell proliferation, migration, invasion, and apoptosis via regulating SPOCK1 expression via miR-613 in GBC cells.

\section{Silence of UCA1 curbed tumor growth in vivo}

Knowing that UCA1 silencing could repress the malignant behaviors of GBC cells in vitro, we further verified the role of UCA1 in GBC in vivo through xenograft assay. We observed that UCA1 silencing evidently reduced tumor growth (volume) and weight in contrast to the control group (Fig. 7A and 7B). Also, UCA1 expression was decreased while miR-613 expression was elevated in mice tumor tissues of the sh-UCA1 group in contrast to the sh-NC group (Fig. 7C and 7D). Besides, the level of SPOCK1 protein was reduced in mice tumor tissues of the sh-UCA1 group that that in the control group (Fig. 7E). Together, these data indicated that UCA1 inhibition reduced tumor growth in vivo.

\section{Discussion}

At present, the specificity of GBC diagnosis is relatively low, and patients with advanced stage have poor progression and high mortality. It is well documented that IncRNAs and miRNAs are potential therapeutic targets for various cancers [23]. Herein, we aimed to explore regulatory mechanism between UCA1 and 
miR-613 in GBC. The results indicated that the expression of UCA1 was upregulated in GBC tissues and cells. Knockdown of UCA1 repressed GBC progression via by downregulation SPOCK1 via sponging miR613.

Convincing evidence showed that UCA1 played a vital role in the progression of various cancers. For example, UCA1 elevation promoted osteosarcoma progression and was correlated with poor prognosis of osteosarcoma [24]. UCA1 could accelerate cell invasion and migration in pancreatic cancer through the hippo pathway [25]. Moreover, reduced UCA1 expression impeded cell invasion and proliferation via upregulating miR-206 in cervical cancer [26]. In the present study, UCA1 was upregulated in GBC tissues and cells. UCA1 knockdown decreased tumor growth in vivo, inhibited cell proliferation, migration, invasion, and promoted cell apoptosis in GBC cells in vitro. Previous study demonstrated that UCA1 promoted GBC advancement through recruiting enhancer of EZH2 to suppress P21 and E-cadherin [27]. Therefore, these data manifested that UCA1 played a carcinogenic role in GBC.

Studies have shown that IncRNAs acts as a sponge for miRNAs and regulate gene expression [28]. In the present study, UCA1 was proved as a sponge for miR-613 in GBC cells. It was reported that miR-613 mimic could repress Daam1 expression in triple-negative breast cancer, which impeded cell invasion and migration [15]. Yang et al. reported that IncRNA HOTAIR enhanced c-met expression via sponging miR613, which facilitated cell the epithelial-mesenchymal transition in retinoblastoma [29]. However, Yang et al. pointed out that miR-613 accelerated colon cancer progression via repressing ATOH1 expression [30]. Also, miR-613 contributed to the advancement of cervical cancer via modulating PTPN9 expression [31]. Herein, miR-613 blocked cell proliferation, migration, invasion, and promoted cell apoptosis in GBC cells. UCA1 overexpression attenuated the effects of miR-613 on the malignant behaviors of GBC cells. These indicated that UCA1 played its function via miR-613 in GBC.

In addition, we discovered that SPOCK1 was a downstream target for miR-613. Increasing evidence suggested that SPOCK1 was upregulated in multiple cancers $[19,21]$. Zhao et al. revealed that SPOCK1 silencing repressed cell invasion and proliferation via impeding the PI3K/AKT pathway [32]. Shu et al. also proved that SPOCK1 was upregulated in GBC tissues, and SPOCK1 overexpression promoted GBC progression via activation of the PI13/AKT pathway [22]. In this study, SPOCK1 expression was elevated in GBC tissues and cells, and forced SPOCK1 expression overturned UCA1 silencing-mediated effects on proliferation, migration, invasion, and apoptosis of GBC cells. Furthermore, SPOCK1 was regulated by the UCA1/miR-613 axis. Therefore, we concluded that UCA1 modulated GBC progression via regulating SPOCK1 expression via miR-613.

\section{Conclusion}

In conclusion, UCA1 silencing impeded GBC advancement through downregulating SPOCK1 via sponging miR-613, implying that UCA1 might be a potential target for the treatment of GBC.

\section{Declarations}


Acknowledgements

Not applicable.

Funding

No funding was received.

\section{Availability of data and materials}

The analyzed data sets generated during the present study are available from the corresponding author on reasonable request.

\section{Authors' contribution}

Both Wei Yu and Yong Dai are in charge the writing, validation, conceptualization, methodology, formal analysis and investigation of this manuscript.

\section{Ethics approval and consent to participate}

The present study was approved by the ethical review committee of West China Hospital, Sichuan University.

\section{Patient consent for publication}

Not applicable.

\section{Competing interests}

The authors declare that they have no competing interests.

\section{References}

1. Wu X-S, Shi L-B, Li M-L, Ding Q, Weng H, Wu W-G, et al. Evaluation of Two Inflammation-Based Prognostic Scores in Patients with Resectable Gallbladder Carcinoma. Annals of Surgical Oncology. 2014;21:449-57.

2. Sharma A, Sharma KL, Gupta A, Yadav A, Kumar A. Gallbladder cancer epidemiology, pathogenesis and molecular genetics: Recent update. World Journal of Gastroenterology. 2017;23:3978-98.

3. Li ML, Zhang Z, Li XG, Ye JY, Wu XS, Tan ZJ, et al. Whole-exome and targeted gene sequencing of gallbladder carcinoma identifies recurrent mutations in the ErbB pathway. Nature Genetics. 2014;46:872-6.

4. lyer MK, Niknafs YS, Malik R, Singhal U, Sahu A, Hosono Y, et al. The landscape of long noncoding RNAs in the human transcriptome. Nature Genetics. 2015;47:199-+. 
5. Li CH, Chen YC. Targeting long non-coding RNAs in cancers: Progress and prospects. International Journal of Biochemistry \& Cell Biology. 2013;45:1895-910.

6. Nie W, Ge HJ, Yang XQ, Sun XJ, Huang H, Tao X, et al. LncRNA-UCA1 exerts oncogenic functions in non-small cell lung cancer by targeting miR-193a-3p. Cancer Letters. 2016;371:99-106.

7. Huang J, Zhou N, Watabe K, Lu Z, Wu F, Xu M, et al. Long non-coding RNA UCA1 promotes breast tumor growth by suppression of p27 (Kip1). Cell Death \& Disease. 2014;5.

8. Jahangiri B, Khalaj-kondori M, Asadollahi E, Sadeghizadeh M. Cancer-associated fibroblasts enhance cell proliferation and metastasis of colorectal cancer SW480 cells by provoking long noncoding RNA UCA1. Journal of Cell Communication and Signaling. 2019;13:53-64.

9. Cai Q, Jin LY, Wang SH, Zhou D, Wang JD, Tang ZH, et al. Long non-coding RNA UCA1 promotes gallbladder cancer progression by epigenetically repressing p21 and E-cadherin expression. Oncotarget. 2017;8:47957-68.

10. Filipowicz W, Bhattacharyya SN, Sonenberg N. Mechanisms of post-transcriptional regulation by microRNAs: are the answers in sight? Nature Reviews Genetics. 2008;9:102-14.

11. Sayed D, Abdellatif M. MICRORNAS IN DEVELOPMENT AND DISEASE. Physiological Reviews. 2011;91:827-87.

12. Kwak PB, Iwasaki S, Tomari Y. The microRNA pathway and cancer. Cancer Science. 2010;101:230915.

13. Cho WCS. MicroRNAs as therapeutic targets and their potential applications in cancer therapy. Expert Opinion on Therapeutic Targets. 2012;16:747-59.

14. Shah MY, Calin GA. MicroRNAs as therapeutic targets in human cancers. Wiley Interdisciplinary Reviews-Rna. 2014;5:537-48.

15. Xiong $H$, Yan $T$, Zhang $W$, Shi $F$, Jiang $X$, Wang $X$, et al. miR-613 inhibits cell migration and invasion by downregulating Daam1 in triple-negative breast cancer. Cellular signalling. 2018;44:33-42.

16. Yu H, Duan P, Zhu H, Rao D. miR-613 inhibits bladder cancer proliferation and migration through targeting SphK1. American journal of translational research. 2017;9:1213-21.

17. Wang J, Yang S, Ge W, Wang Y, Han C, Li M. MiR-613 suppressed the laryngeal squamous cell carcinoma progression through regulating PDK1. Journal of cellular biochemistry. 2018;119:5118-25.

18. Ahel D, Horejsi Z, Wiechens N, Polo SE, Garcia-Wilson E, Ahel I, et al. Poly(ADP-ribose)-Dependent Regulation of DNA Repair by the Chromatin Remodeling Enzyme ALC1. Science. 2009;325:1240-3.

19. Chen Q, Yao Y-t, Xu H, Chen Y-b, Gu M, Cai Z-k, et al. SPOCK1 promotes tumor growth and metastasis in human prostate cancer. Drug Design Development and Therapy. 2016;10:2311-21.

20. Ma L-J, Wu W-J, Wang Y-H, Wu T-F, Liang P-I, Chang IW, et al. SPOCK1 Overexpression Confers a Poor Prognosis in Urothelial Carcinoma. Journal of Cancer. 2016;7:467-76.

21. Zhang J, Zhi X, Shi S, Tao R, Chen P, Sun S, et al. SPOCK1 is up-regulated and promotes tumor growth via the PI3K/AKT signaling pathway in colorectal cancer. Biochemical and Biophysical Research Communications. 2017;482:870-6. 
22. Shu YJ, Weng H, Ye YY, Hu YP, Bao RF, Cao Y, et al. SPOCK1 as a potential cancer prognostic marker promotes the proliferation and metastasis of gallbladder cancer cells by activating the PI3K/AKT pathway. Mol Cancer. 2015;14:12.

23. Mahmoudian-Sani MR, Jalali A, Jamshidi M, Moridi H, Alghasi A, Shojaeian A, et al. Long NonCoding RNAs in Thyroid Cancer: Implications for Pathogenesis, Diagnosis, and Therapy. Oncology Research and Treatment. 2019;42:136-42.

24. Li W, Xie P, Ruan WH. Overexpression of IncRNA UCA1 promotes osteosarcoma progression and correlates with poor prognosis. Journal of Bone Oncology. 2016;5:80-5.

25. Zhang $M$, Zhao $Y$, Zhang $Y$, Wang D, Gu S, Feng W, et al. LncRNA UCA1 promotes migration and invasion in pancreatic cancer cells via the Hippo pathway. Biochimica et biophysica acta Molecular basis of disease. 2018;1864:1770-82.

26. Yan Q, Tian Y, Hao F. Downregulation of IncRNA UCA1 inhibits proliferation and invasion of cervical cancer cells through miR-206 expression. Oncology research. 2018.

27. Cai Q, Jin L, Wang S, Zhou D, Wang J, Tang Z, et al. Long non-coding RNA UCA1 promotes gallbladder cancer progression by epigenetically repressing p21 and E-cadherin expression. Oncotarget. 2017;8:47957-68.

28. Tay Y, Rinn J, Pandolfi PP. The multilayered complexity of ceRNA crosstalk and competition. Nature. 2014;505:344-52.

29. Yang G, Fu Y, Lu X, Wang M, Dong H, Li Q. LncRNA HOTAIR/miR-613/c-met axis modulated epithelialmesenchymal transition of retinoblastoma cells. Journal of cellular and molecular medicine. 2018;22:5083-96.

30. Yang X, Zhang L, Song X, He W, Zhang D, Lu Q, et al. MicroRNA-613 promotes colon cancer cell proliferation, invasion and migration by targeting ATOH1. Biochemical and biophysical research communications. 2018;504:827-33.

31. Li WT, Wang BL, Yang CS, Lang BC, Lin YZ. MiR-613 promotes cell proliferation and invasion in cervical cancer via targeting PTPN9. European review for medical and pharmacological sciences. 2018;22:4107-14.

32. Zhao P, Guan H-T, Dai Z-J, Ma Y-G, Liu X-X, Wang X-J. Knockdown of SPOCK1 Inhibits the Proliferation and Invasion in Colorectal Cancer Cells by Suppressing the PI3K/Akt Pathway. Oncology research. 2016;24:437-45.

\section{Figures}


A

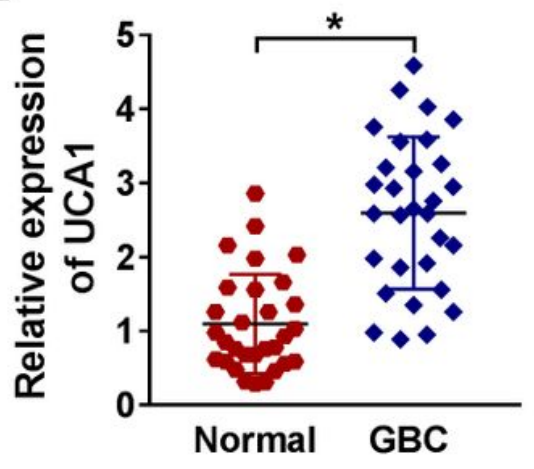

D

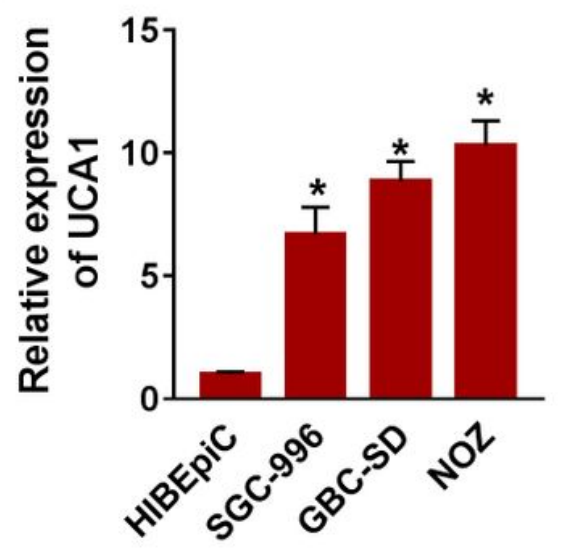

B

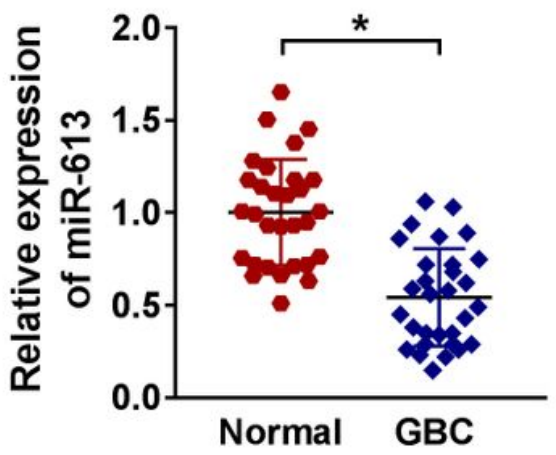

$\mathbf{E}$
C

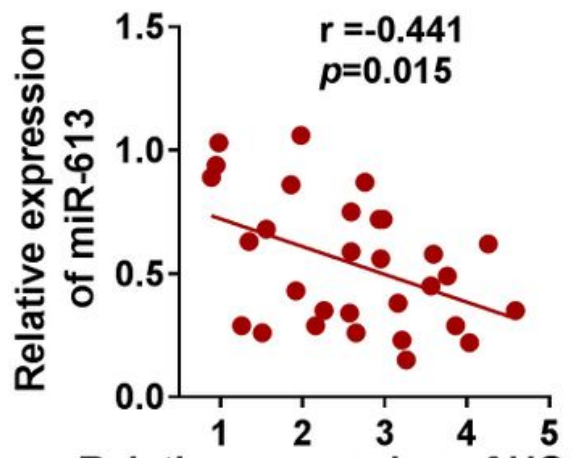

Relative expression of UCA1

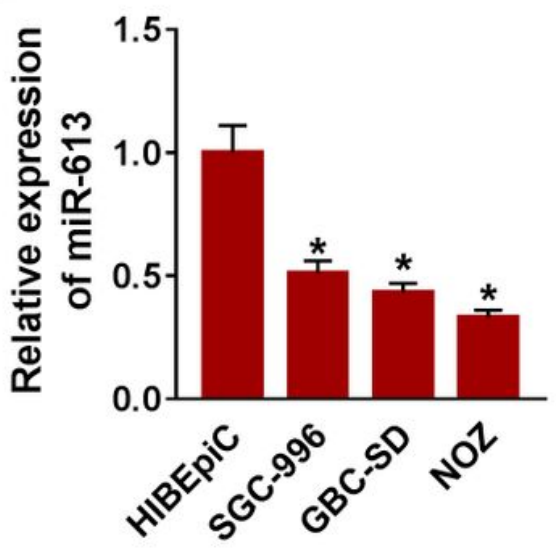

Figure 1

Expression levels of UCA1 and miR-613 in GBC tissues and cells. (A and B) QRT-PCR was performed to analyze the expression levels of UCA1 and miR-613 in GBC tissues and adjoining normal tissues. (C) The correlation between UCA1 and miR-613 in GBC tissues was determined via Pearson's correction analysis. (D and E) QRT-PCR was executed to assess the expression levels of UCA1 and miR-613 in GBC cells (SGC996, GBC-SD, and NOZ) and the HIBEpiC cells. ${ }^{\star P} \otimes 0.05$. 
A

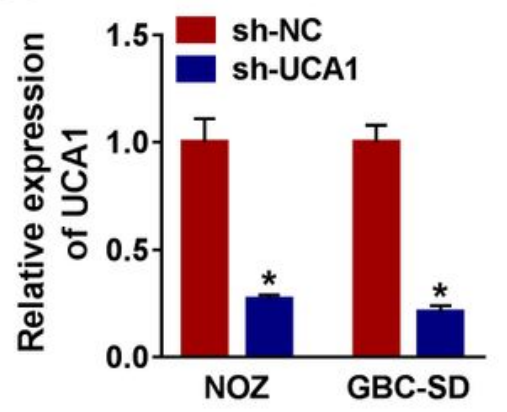

D

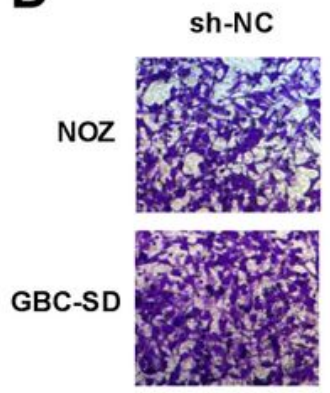

sh-UCA1

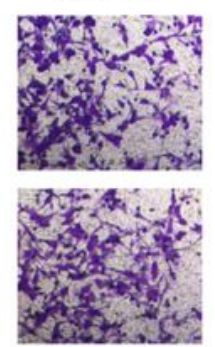

E

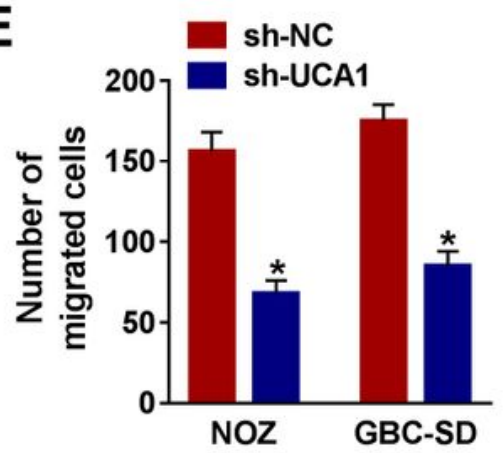

B

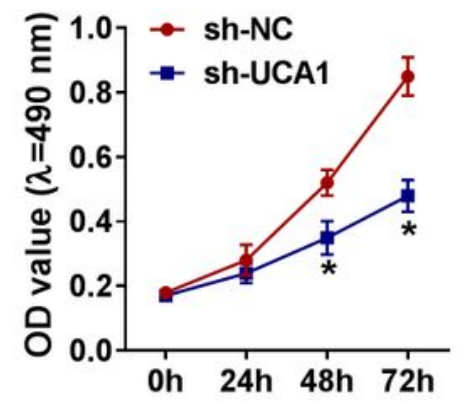

F

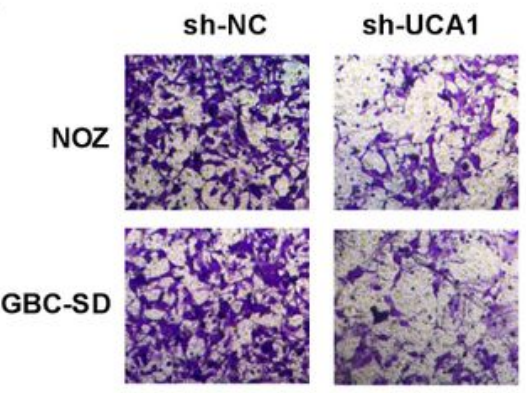

G

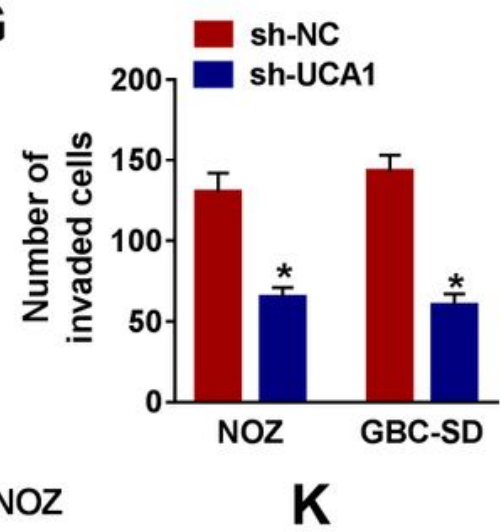

J

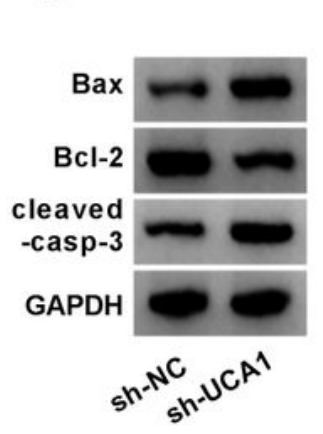

C

GBC-SD

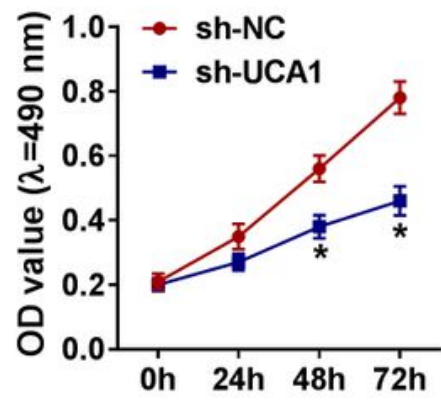

H

NOZ

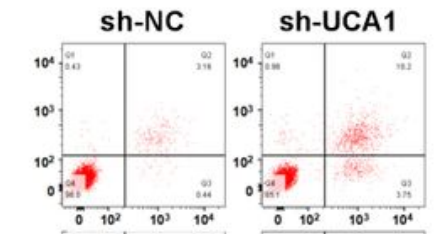

GBC-SD -
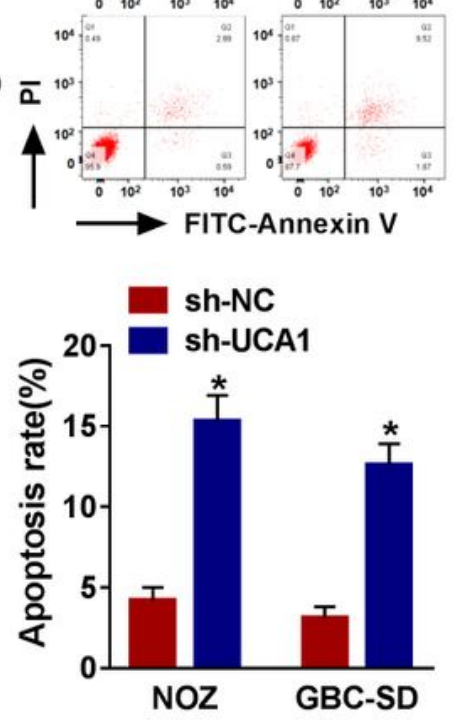

OCUG

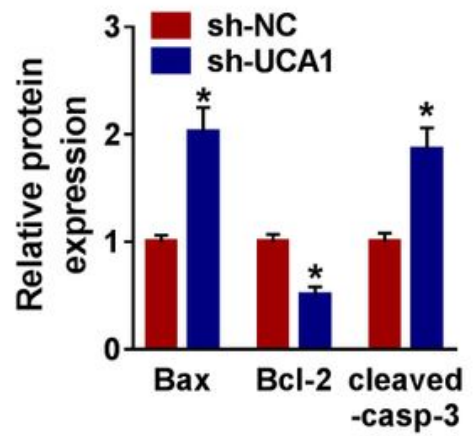

Figure 2

Effects of UCA1 silencing on the proliferation, migration, invasion, and apoptosis of GBC cells. NOZ and GBC-SD cells were transfected with sh-UCA1 or sh-NC. (A) The expression levels of UCA1 in NOZ and GBC-SD cells were detected by qRT-PCR. (B-I) The proliferation (B and C), migration (D and E), invasion ( $F$ and $\mathrm{G}$ ), and apoptosis ( $\mathrm{H}$ and I) of NOZ and GBC-SD cells were determined with MTT, transwell, or flow 
cytometry assays. ( $\mathrm{J}$ and $\mathrm{K}$ ) The levels of Bax, cleaved-casp-3, and Bcl-2 in NOZ and GBC-SD cells were detected by using western blot analysis. *P®0.05.

A

WT-UCA1 5' UAACCCACCUUACGACAUUCCA 3'

miR-613 3' CCGUUUCUUCCUUGUAAGG 5'

MUT-UCA1 5' UAACCCACCUUACGUGUAAGGA 3'
B

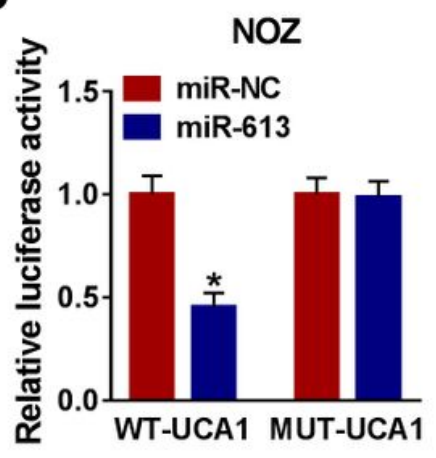

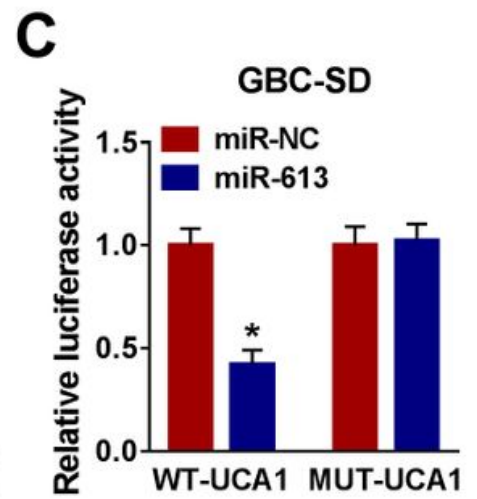

D

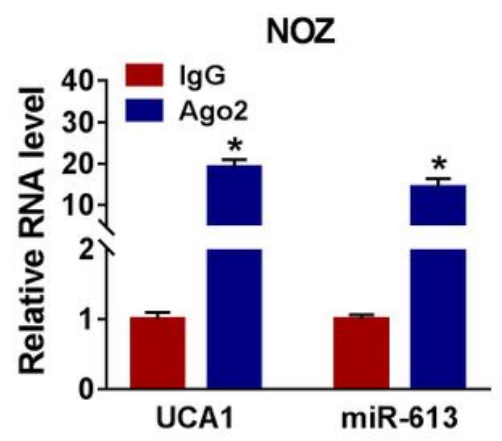

G

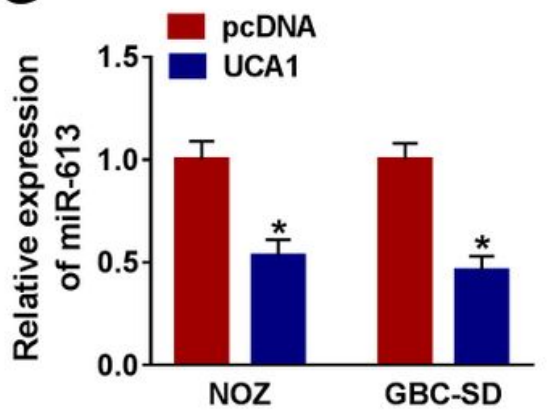

$\mathbf{E}$
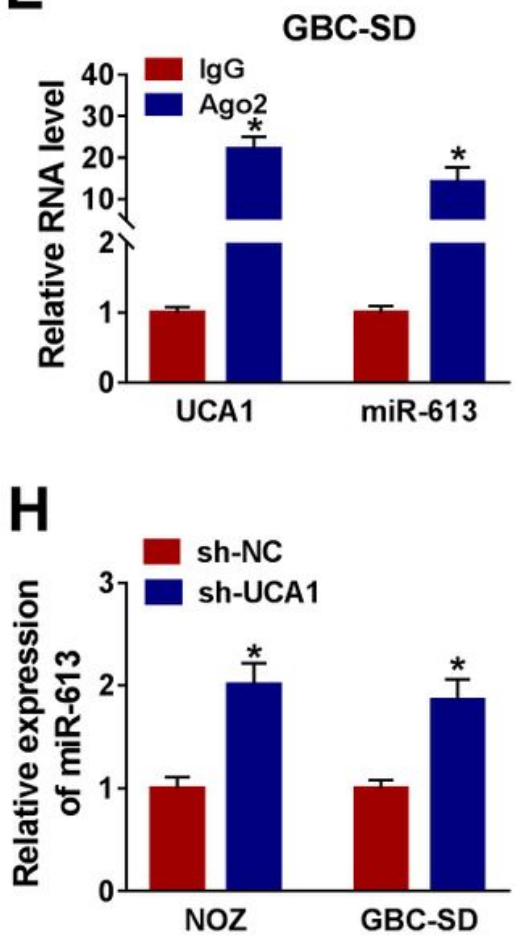

$\mathbf{F}$

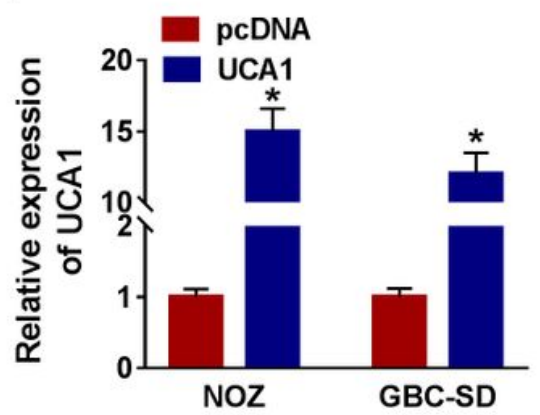

\section{Figure 3}

UCA1 served as a sponge for miR-613 in GBC cells. (A) Predicted binding sites of miR-613 in UCA1 were displayed. (B and C) Dual-luciferase reporter assays were performed to determine the luciferase activities of WT-UCA1 and MUT-UCA1 in NOZ and GBC-SD cells cotransfected with miR-613 or miR-NC. (D and E) RIP assays were employed to verify the relationship between miR-613 and UCA1 in NOZ and GBC-SD cells. (F) The expression of UCA1 in NOZ and GBC-SD cells transfected with UCA1 or pcDNA was assessed by qRT-PCR. ( $G$ and $H$ ) Influence of UCA1 enhancement or repression on the expression of miR613 was evaluated with qRT-PCR. *Pख0.05. 

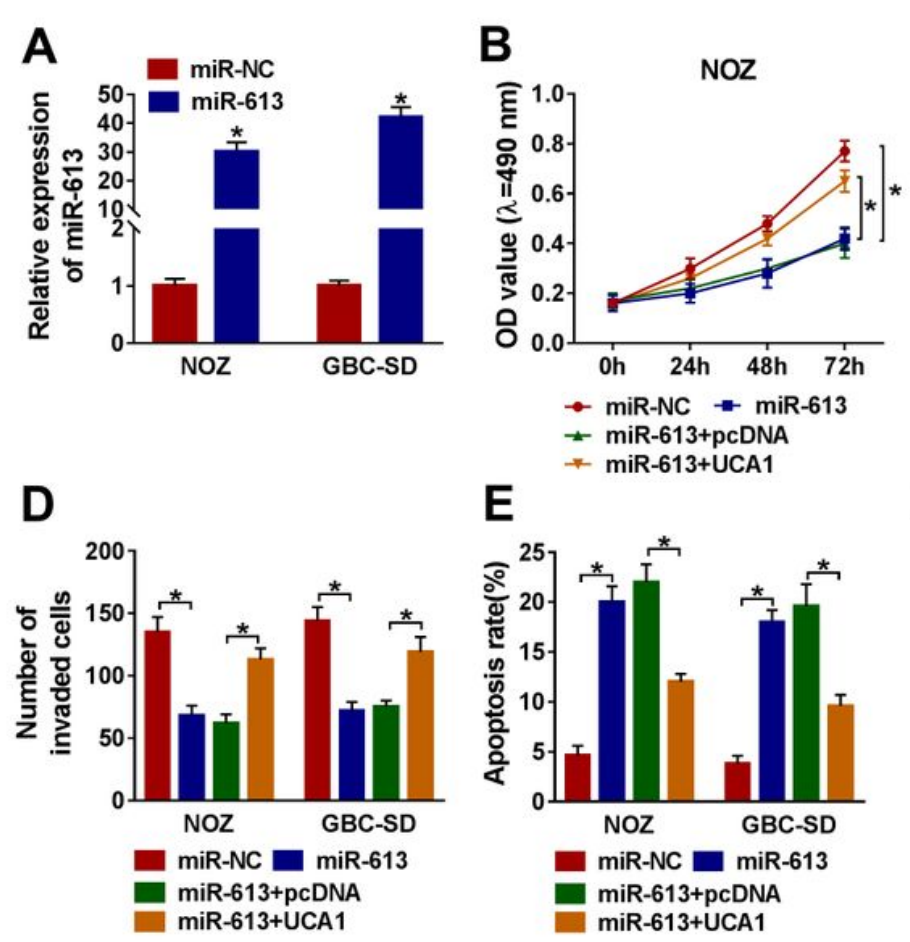

F
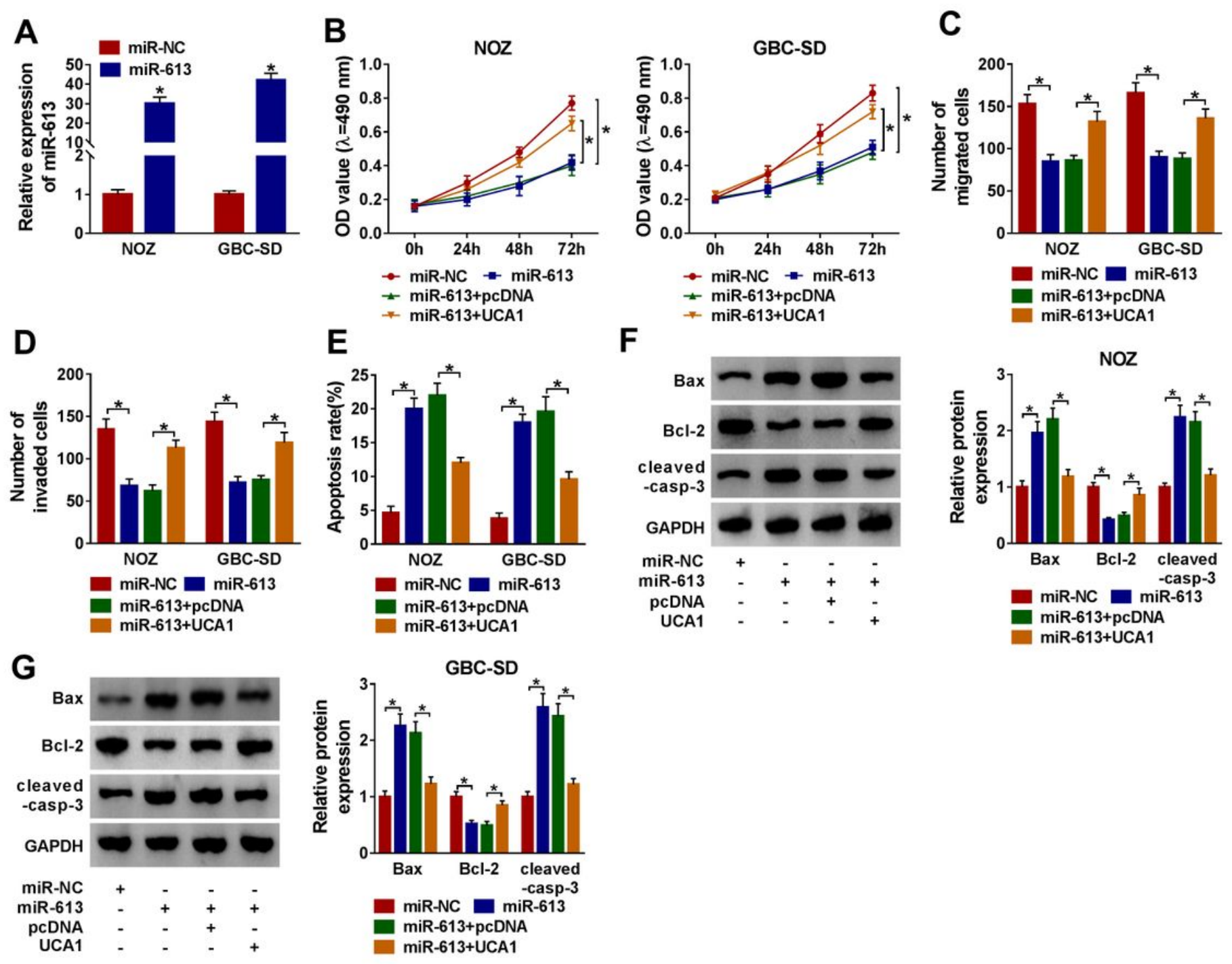

Figure 4

UCA1 upregulation reversed the impacts of miR-613 elevation on the proliferation, migration, invasion, and apoptosis of GBC cells. (A) Expression of miR-613 in NOZ and GBC-SD cells transfected with miR613 or miR-NC. (B-E) Effects of UCA1 overexpression on the proliferation (B), migration (C), invasion (D), apoptosis (E) of miR-613-elevated NOZ and GBC-SD cells were assessed with MTT, transwell, or flow cytometry assays. ( $F$ and $G$ ) Impacts of UCA1 overexpression on the levels of Bax, cleaved-casp-3, and Bcl-2 of miR-613-elevated NOZ and GBC-SD cells were examined through western blot analysis. *P囚0.05. 
B

SPOCK1 3'UTR-WT
5' GGUCAUCAUCUCUACAUUCCU 3'
miR-613
3' CCGUUUCUUCCUUGUAAGGA 5'

SPOCK1 3'UTR-MUT 5' GGUCAUCAUCUCUUGUAAGGU 3'
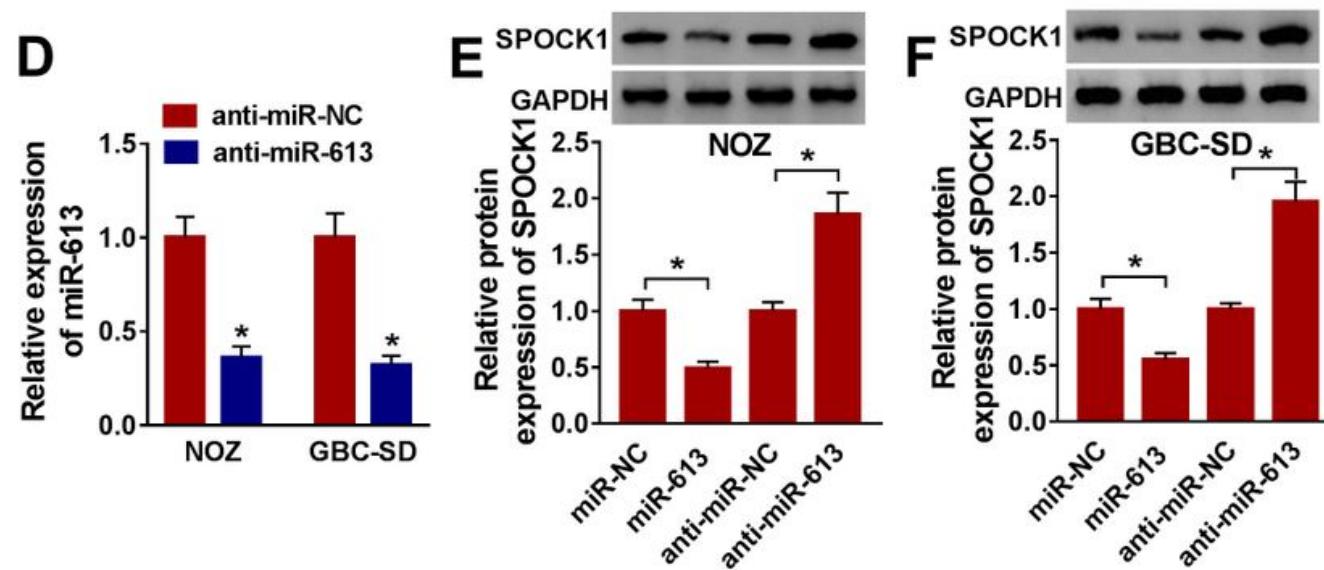

H

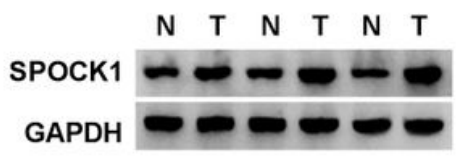

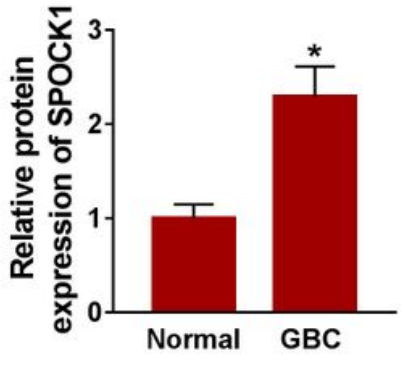

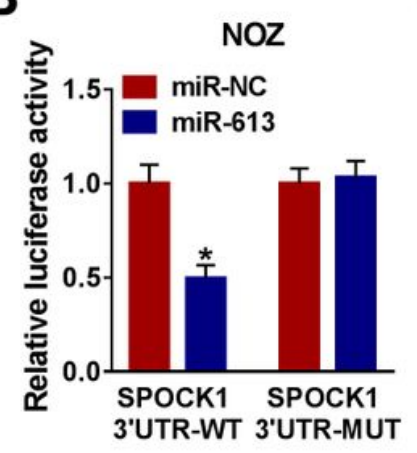

C

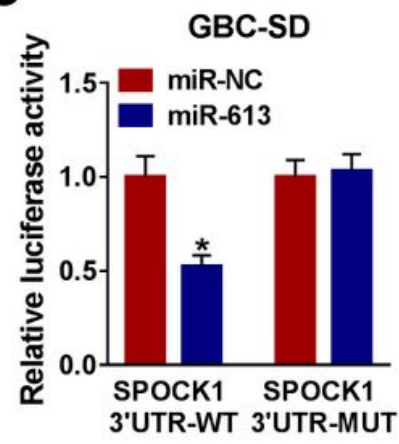

G
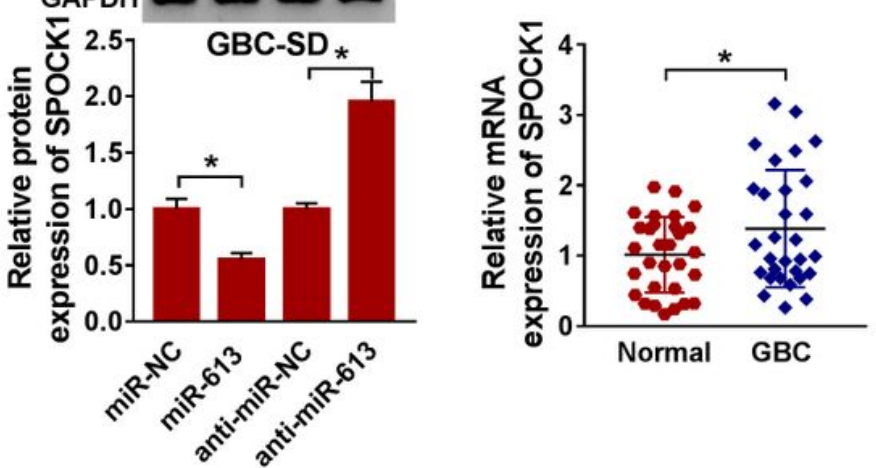

I

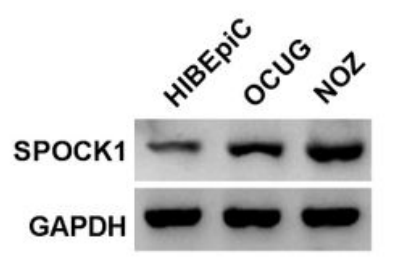

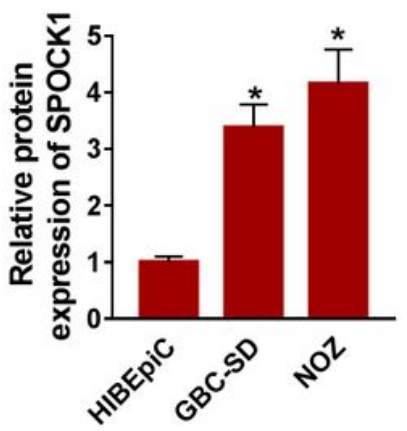

Figure 5

SPOCK1 acted as a target for miR-613. (A) The binding sites between miR-613 and SPOCK1 were predicted with the starBase2.0 database. (B and C) The luciferase intensities of SPOCK1 3'UTR-WT and SPOCK1 3'UTR-MUT in NOZ and GBC-SD cells co-transfected with miR-613 or miR-NC were detected via dual-luciferase reporter assays. (D) Expression level of miR-613 in NOZ and GBC-SD cells transfected with anti-miR-613 or anti-miR-NC was examined by qRT-PCR. (E and F) Western blot analysis was performed to evaluate the influence of miR-613 introduction or silencing on the expression levels of SPOCK1 protein. (G-I) The expression levels of SPOCK1 mRNA and protein in GBC tissues and cells were detected with qRT-PCR or western blot analysis. *Pख0.05. 

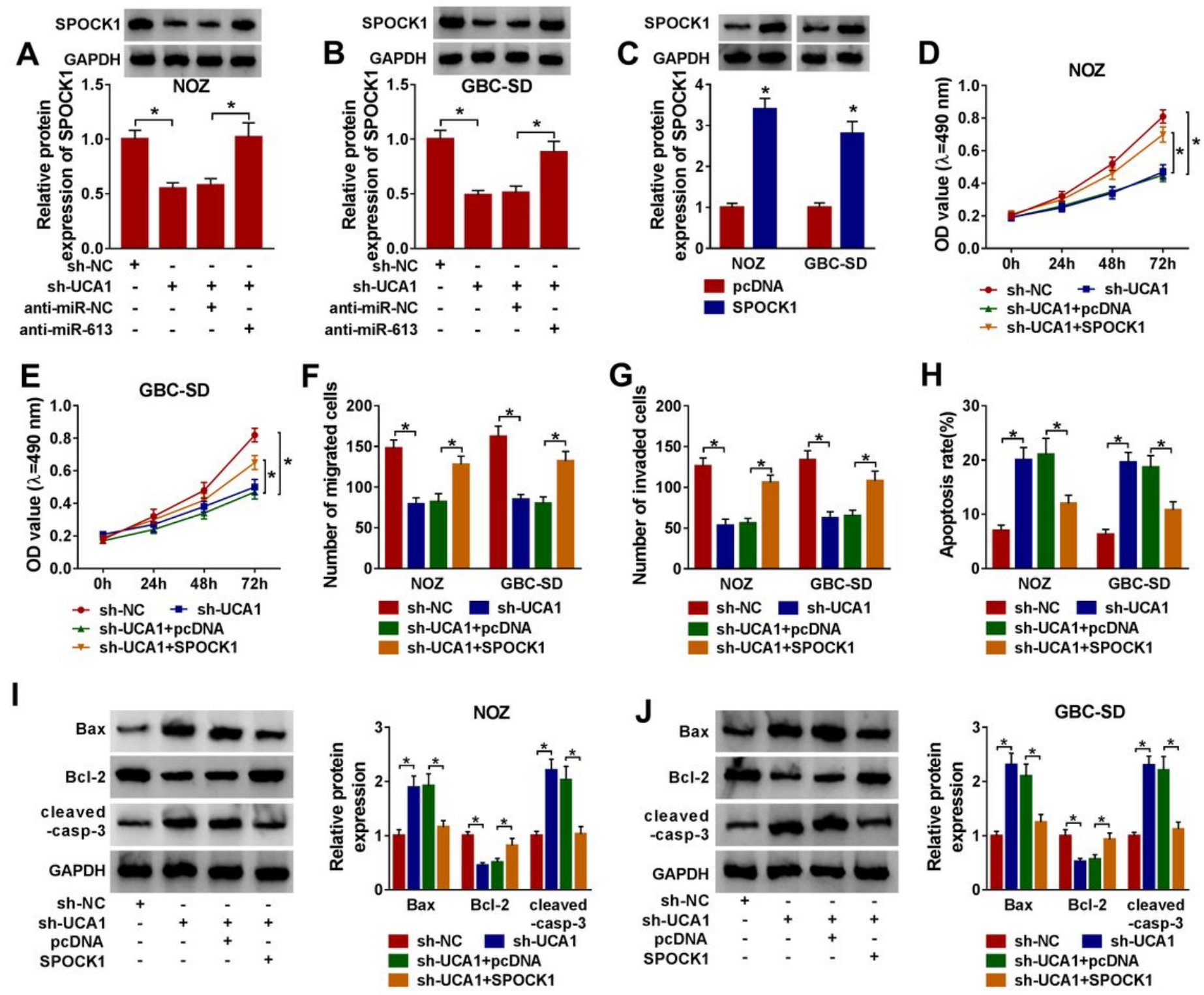

Figure 6

SPOCK1 elevation restored UCA1 silencing-mediated effects on the proliferation, migration, invasion, and apoptosis of GBC cells. (A and B) Effect of miR-613 knockdown on the protein expression levels of SPOCK1 of UCA1-silenced NOZ and GBC-SD cells was determined via western blot analysis. (C) Expression levels of SPOCK1 in NOZ and GBC-SD cells transfected with pCDNA or SPOCK1 were detected with western blot analysis. (D-H) Influence of SPOCK1 overexpression on the proliferation, migration, invasion, and apoptosis of UCA1-silenced NOZ and GBC-SD cells was analyzed through MTT, transwell, or flow cytometry assays. (I and J) Impacts of SPOCK1 upregulation on the levels of Bax, cleaved-casp-3, and Bcl-2 of UCA1-suppressed NOZ and GBC-SD cells were evaluated via western blot analysis. *P囚0.05. 
A

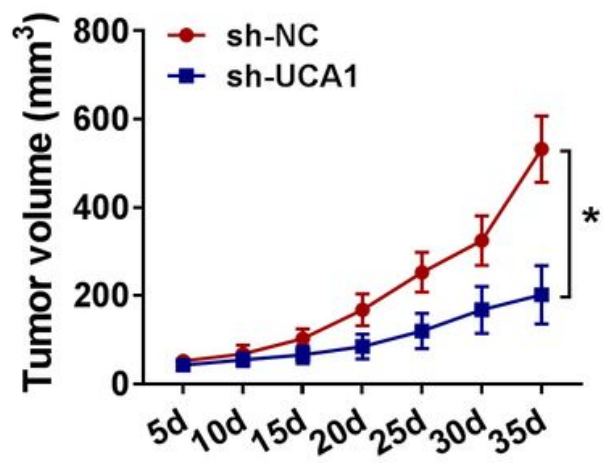

D

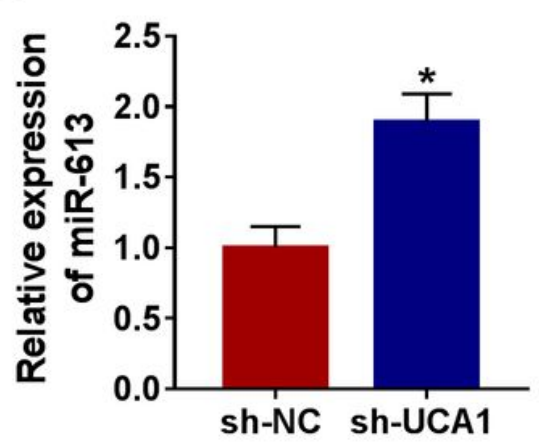

B

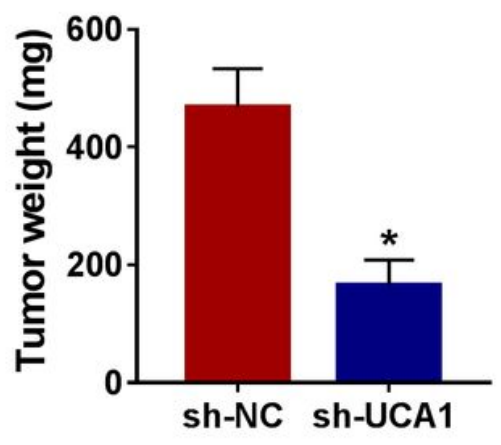

$\mathbf{E}$

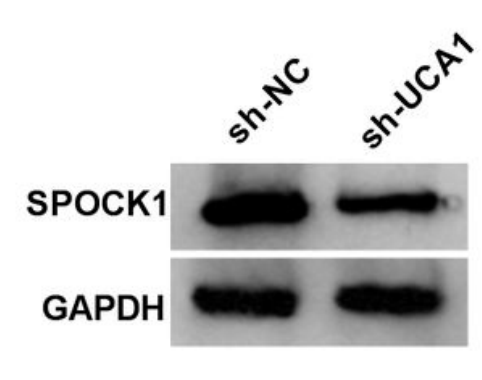

C

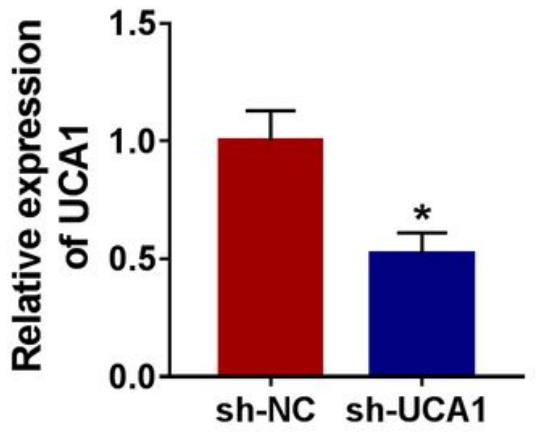

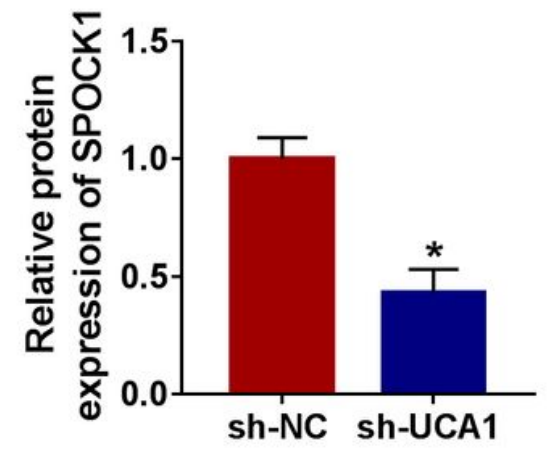

Figure 7

UCA1 silencing impeded tumor growth in vivo. (A) Tumor volume was measured every 5 days until day 35. (B) Mice were killed to assess tumor weight on day 35 after injection. (C-E) The expression levels of UCA1, miR-613, and SPOCK1 protein in tumor tissues of mice of the sh-UCA1 and sh-NC groups were examined via qRT-PCR or western blot analysis. *P囚0.05. 